\title{
Improvement of Throughput Prediction Scheme Considering Terminal Distribution in Multi-Rate WLAN Considering Both CSMA/CA and Frame Collision**
}

\author{
Ryo HAMAMOTO $^{\dagger * a)}$, Chisa TAKANO ${ }^{\dagger b)}$, Hiroyasu OBATA $^{\dagger c)}$, and Kenji ISHIDA $^{\dagger \mathrm{d})}$, Members $^{\text {. }}$
}

SUMMARY Wireless Local Area Networks (WLANs) based on the IEEE 802.11 standard have been increasingly used. Access Points (APs) are being established in various public places, such as railway stations and airports, as well as private residences. Moreover, the rate of public WLAN services continues to increase. Throughput prediction of an AP in a multi-rate environment, i.e., predicting the amount of receipt data (including retransmission packets at an AP), is an important issue for wireless network design. Moreover, it is important to solve AP placement and selection problems. To realize the throughput prediction, we have proposed an AP throughput prediction method that considers terminal distribution. We compared the predicted throughput of the proposed method with a method that uses linear order computation and confirmed the performance of the proposed method, not by a network simulator but by the numerical computation. However, it is necessary to consider the impact of CSMA/CA in the MAC layer, because throughput is greatly influenced by frame collision. In this paper, we derive an effective transmission rate considering CSMA/CA and frame collision. We then compare the throughput obtained using the network simulator NS2 with a prediction value calculated by the proposed method. Simulation results show that the maximum relative error of the proposed method is approximately $6 \%$ and $15 \%$ for UDP and TCP, respectively, while that is approximately $17 \%$ and $21 \%$ in existing method. key words: wireless LAN, access point, multi-rate, throughput prediction, network design

\section{Introduction}

With the rapid growth of wireless communication technologies, Wireless Local Area Networks (WLANs) based on the IEEE802.11 standard [2] have become increasingly popular. The number of public WLAN service areas has been increasing as WLAN Access Points (APs) are widely implemented, e.g., in railway stations and airports [3]. In addition, even a smartphone can be used as an AP through tethering technology. Therefore, Internet connections through WLANs have become common. In WLANs based on the IEEE 802.11 standard, APs and terminals use multi-rate

Manuscript received January 8, 2016.

Manuscript revised May 17, 2016.

Manuscript publicized August 24, 2016.

${ }^{\dagger}$ The authors are with Graduate School of Information Sciences, Hiroshima City University, Hiroshima-shi, 731-3194 Japan.

* Presently, with Security Research Laboratories NEC Corporation, Japan.

${ }^{* *}$ An earlier version of this paper was presented at the 8th International Workshop on ASON 2015 [1].

a) E-mail: ryo@net.info.hiroshima-cu.ac.jp

b) E-mail: takano@hiroshima-cu.ac.jp

c)E-mail: obata@hiroshima-cu.ac.jp

d)E-mail: ishida@hiroshima-cu.ac.jp

DOI: 10.1587/transinf.2016PAP0019 transmission for effective communication because each terminal has a different communication environment. In multirate transmission technology, the transmission rate is set relative to the electric wave environment between the AP and a wireless terminal. A terminal with a good electric wave environment can connect at high transmission rates, whereas those with poor environments realize low transmission rates. As a result, each terminal can obtain better throughput depending on the radio wave environments.

The AP selection [4] and placement problems [5] have been investigated relative to wireless network design. The AP throughput (the quantity of data that an AP can receive including retransmission packets) can be used as a metric for these problems. However, it is necessary to estimate AP throughput in advance. Several studies have proposed and evaluated AP throughput prediction methods [6]-[9]. For example, [9] explains that in a multi-rate environment, AP throughput is equal to the harmonic average of the transmission rates of the terminals connected to the AP. Moreover, an analytic proof that the harmonic average of the transmission rate is the upper limit of the throughput of the AP has been published [9]. In addition, the value predicted by [9] is nearly equal to the simulation value (true value) obtained by the network simulator NS2 [10] in an IEEE802.11b/a WLAN environment and UDP flow case. Generally, terminal transmission rate selection methods in real machines are highly dependent on the radio wave environment. In this paper, the throughput between an AP and each terminal is calculated by assuming the transmission rate steps for the distance between the AP and the terminal [11]-[13] (see Fig. 1). Thus, AP throughput is predicted by the harmonic average of calculated throughput for all terminals and the computational complexity is linear with the number of terminals. The existing method [9] can predict AP throughput with high precision under assumption that the transmission rates of all terminals are known.

We have previously proposed a high-speed AP throughput prediction method that considers terminal distribution (placement) [14] in a multi-rate WLAN environment. Moreover, we have determined that the predicted value of [14] is nearly equal to the predicted value reported by [9]. In addition, [14] have compared the proposed method with an existing prediction method relative to the calculation cost. [14] has performed a basic evaluation and a performance comparison, not by a network simulator but by the numer- 
Table 1 Relationship between distance and theoretical transmission rate (IEEE802.11a/g) [12].

\begin{tabular}{|l|l|l|l|l|l|l|l|l|}
\hline Index $i$ & 1 & 2 & 3 & 4 & 5 & 6 & 7 & 8 \\
\hline Threshold of distance $d_{i}$ from AP m & 5 & 7 & 9 & 20 & 25 & 40 & 50 & 60 \\
\hline Transmission rate $b_{i}$ Mbps & 54 & 48 & 36 & 24 & 18 & 12 & 9 & 6 \\
\hline
\end{tabular}

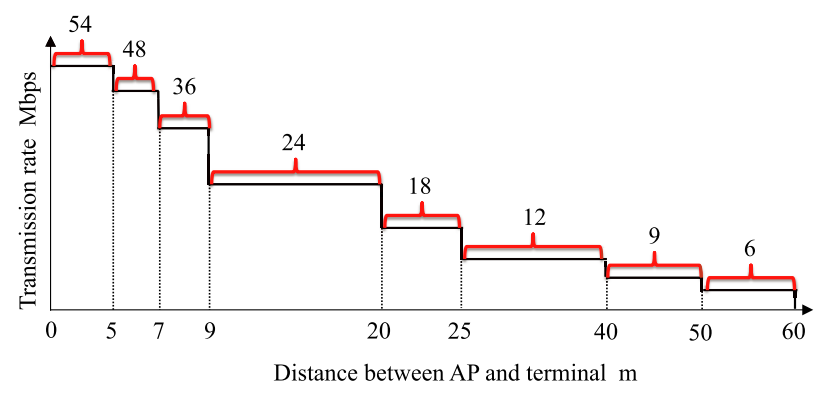

Fig. 1 Stepped transmission rate.

ical computation. However, it is necessary to consider the impact of CSMA/CA in the MAC layer, because throughput is greatly influenced by frame collision. In this paper, firstly, we derive an effective transmission rate considering CSMA/CA and frame collision. We then compare the throughput obtained with the network simulator NS2 to the prediction value calculated by both the proposed method and the existing method [9]. In the simulation, UDP [1] and TCP are used as the transport protocols. The results show that the maximum relative error of the proposed method is approximately $6 \%$ and $15 \%$ for UDP and TCP, respectively, while that is approximately $17 \%$ and $21 \%$ in existing method.

\section{Multi-Rate Transmission of IEEE802.11 and Exist- ing Throughput Prediction Method}

Most IEEE 802.11b/a/g WLANs [2] employ multi-rate transmission. In IEEE $802.11 \mathrm{~b}$, the transmission rates are 11, 5.5, 2, and $1 \mathrm{Mbps}$, whereas IEEE 802.11a/g offers 54, 48, 36, 24, 18, 12, 9, and 6 Mbps. These protocols specify multiple transmission rates, and the appropriate transmission rate can be selected so as not to exceed a certain bit error or frame error rate [15]. The appropriate transmission rate is usually selected by the original vendor algorithm ${ }^{\dagger}$. However, some products allow the transmission rate to be selected manually. For example, in transmission rate control [16], if the electric wave environment worsens and highspeed transmission cannot be maintained, the AP can reduce the transmission rate.

In [12], one of the stepped transmission rates is selected according to distance (Table 1). Table 1 summarizes the distance $d_{i}$, which is the maximum distance between each terminal and the AP without bit error, and the theoretical value of the transmission rate $b_{i}$ is selected by the AP, as per IEEE $802.11 \mathrm{a} / \mathrm{g}$. Index $i(i=1,2, \cdots, 8)$ is allocated in ascending order of distance from the AP. If the distance between the AP and the terminal is less than $5 \mathrm{~m}$, the trans-

\footnotetext{
${ }^{\dagger}$ Generally, the algorithm is closed for the public.
}

mission rate of $i=1$ is selected. If the distance is greater than $60 \mathrm{~m}$, the terminal cannot connect to the AP. In this paper, we use this selection algorithm for transmission rate.

From [9], it is known that AP throughput $\theta$ can be estimated using Eq. (1) in a multi-rate environment.

$$
\theta=n_{\text {cnt }}\left(\sum_{j=1}^{N}\left(b_{j}\right)^{-1}\right)^{-1}
$$

In Eq. (1), $N$ denotes the number of terminals in the system and $n_{c n t}(\leq N)$ denotes the number of terminals connected to the AP with transmission rates greater than $0 \mathrm{bps}$. $b_{j}$ is the transmission rate of the $j$-th terminal connected to the AP, which varies according to the distance between the $\mathrm{AP}$ and the terminal. A transmission rate of 0 bps is dropped from the denominator of Eq. (1). If the positional information of each terminal is known by the AP, the throughput value predicted by Eq. (1) is very accurate. From Eq. (1), as the number of terminals increases, the computational complexity in terms of the sum in the denominator increases linearly. This time complexity has a significant impact on throughput predictions when real-time response is required. For example, when a terminal uses real-time transfer, it must be able to determine that the bandwidth is sufficient for the given application.

\section{Throughput Prediction Method Considering Termi- nal Distribution}

This section presents an overview of a throughput prediction method that considers terminal distribution [14]. Note that the AP occupies the center of a circular radio transmission area.

3.1 Overview of the Throughput Prediction Method Considering Terminal Distribution

We define $n_{i}$ as the number of terminals communicating at transmission rate $b_{i}(i=1,2, \cdots, 8)$ using the data presented in Table 1. Using $n_{i}$, Eq. (1) can be rewritten as Eq. (2):

$$
n_{\text {cnt }}\left(\sum_{j=1}^{N}\left(b_{j}\right)^{-1}\right)^{-1}=n_{c n t}\left(\sum_{i=1}^{8} n_{i} b_{i}^{-1}\right)^{-1} \text {. }
$$

From Eq. (2), the computational complexity of the proposed method is $O(1)$. Thus, the time required for throughput prediction is constant regardless of the number of terminals. In Eq. (2), $n_{i}$ and $n_{\text {cnt }}$ can be represented as follows:

$$
\begin{aligned}
& n_{i}=p_{i} N, \\
& n_{\text {cnt }}=\sum_{i=1}^{8} p_{i} N .
\end{aligned}
$$


In Eqs. (3) and (4), $p_{i}$ implies the ratio of terminals with transmission rate $b_{i}$. In other words, $p_{i}$ is the probability that the terminal uses $b_{i}$. Thus, Eq. (2) can be rewritten as follows:

$$
\theta_{\text {pred }}=\sum_{i=1}^{8} p_{i}\left(\sum_{i=1}^{8} p_{i} b_{i}^{-1}\right)^{-1} .
$$

From Eq. (5), the proposed method can predict the throughput from $p_{i}$ with cost $O(1)$. This paper refers to the throughput predicted by Eq. (5) as the prediction throughput value $\left(\theta_{\text {pred }}\right)$. Next, we discuss a method to predict $p_{i}$ with cost $O(1)$. This study assumes that the terminals are placed according to two-dimensional normal or uniform distribution. The two-dimensional normal distribution has terminals concentrated near the AP. When the AP is placed in a landmark location, such as an airborne balloon used as an AP in an emergency communication network during a large-scale natural disaster [17], the AP will attract many users. As a result, the distribution of users follows a normal distribution. If nodes are dropped vertically from a certain point midair, their spatial distribution will result in a two-dimensional normal distribution [18], [19]. Uniform distribution is the most common user distribution and assumes that terminals are widely distributed in the system. Note that a proposal for throughput prediction considering other terminal distribution is the future work.

\subsection{Throughput Prediction Method Based on the Target} Problem

Here we present an overview of the two-dimensional target problem [20] and describe the throughput prediction method based on the target problem. In this method, the network structure (terminal distribution) is limited to normal distribution. We consider a common situation in which many terminals are placed near the AP. The terminals can be considered equivalent to the arrows that an archer fires at a target. The hit points have a probabilistic characteristic. The twodimensional target problem considers the distribution of hit points. Random variables $X_{i}(i=1,2, \cdots, n)$ are independent of each other, and the normal distribution has variance $\sigma_{i}^{2}$ and average $\mu$. Random variable $Z$ is defined by Eq. (6):

$$
Z=\sum_{i=1}^{n}\left(\frac{X_{i}-\mu_{i}}{\sigma_{i}}\right)^{2} .
$$

$Z$ has $\chi^{2}$ distribution with $n$ flexibility. This implies that the sum of the square of independent random variables that follow standard normal distribution $N(0,1)$ has $\chi^{2}$ distribution. In other words, the distribution of the square sum of the distance between the hit points and the origin of the space has $\chi^{2}$ distribution. In the two-dimensional target problem, the distribution of distance is important. We consider the $\chi$ distribution as the square root distribution of the $\chi^{2}$ distribution, i.e., the square root of the squared sum of distances from the origin to the hit point. Thus, the distribution of the

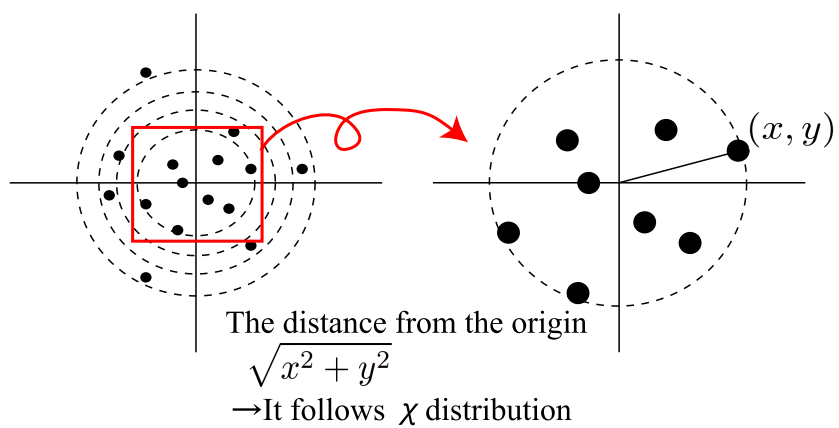

Fig. 2 Relationship between distance from the origin and $\chi$ distribution in the two-dimensional target problem.

distance from the origin indicates a $\chi$ distribution if flexibility $n$ yields each component of the Cartesian coordinates (see Fig. 2). Therefore, in the two-dimensional target problem, the bullet hit probability takes $\chi$ distribution if the size of the target is known and the neighboring distribution of the hit points is a normal distribution. For example, we assume a target with radius $r$ whose origin is the center of a twodimensional plane. Hit probability $F(r)$ has $\chi$ distribution with flexibility of 2 when the neighboring distribution of hit points follows two-dimensional $N\left(0, \sigma^{2}\right)$. In other words, it follows a Rayleigh distribution expressed as follows:

$$
F(r)=1-\exp \left(-\frac{r^{2}}{2 \sigma^{2}}\right) .
$$

Moreover, the probability that the hit point is out of the target (miss probability) $Y(r)$ is expressed by the complementary distribution of Eq. (7) $(1-F(r))$ :

$$
Y(r)=\exp \left(-\frac{r^{2}}{2 \sigma^{2}}\right)
$$

From Eq. (8), we can estimate the number of terminals using each transmission rate. As a result, this method can predict the throughput with $O(1)$ time complexity. We assume that the AP is placed at the origin of the system field and that the terminals are distributed according to two-dimensional $N\left(0, \sigma^{2}\right)$. We consider the probability of a terminal being outside the area of radius $d_{i-1}$ but within the area of radius $d_{i}\left(>d_{i-1}\right)$. In Fig. 3, this is the probability that the terminal lies in the colored area. From Eq. (8), the probability that the terminal is outside the area of radius $d_{i-1}$ and $d_{i}$ is $Y\left(d_{i-1}\right)$ and $Y\left(d_{i}\right)$, respectively. Thus, the ratio of the number of terminals $p_{i}$ whose transmission rate is $b_{i}$ is defined by Eq. (9):

$$
p_{i}=Y\left(d_{i-1}\right)-Y\left(d_{i}\right) \quad(i=1,2, \cdots, 8) .
$$

By substituting $p_{i}$ for Eq. (5), the proposed method can predict throughput. In addition, this method can be performed in $O(1)$ time.

\subsection{Throughput Prediction Method Based on Area Ratio}

Next, we describe a throughput prediction method based on 


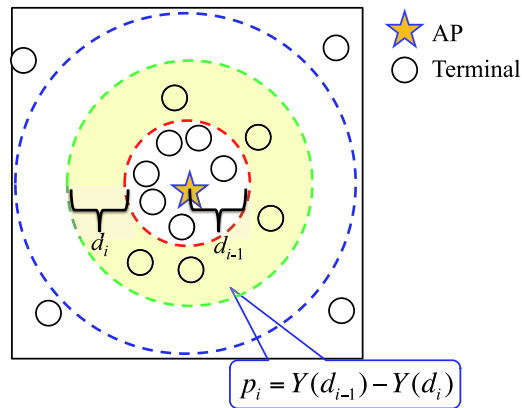

Fig. 3 Throughput prediction based on the target problem.

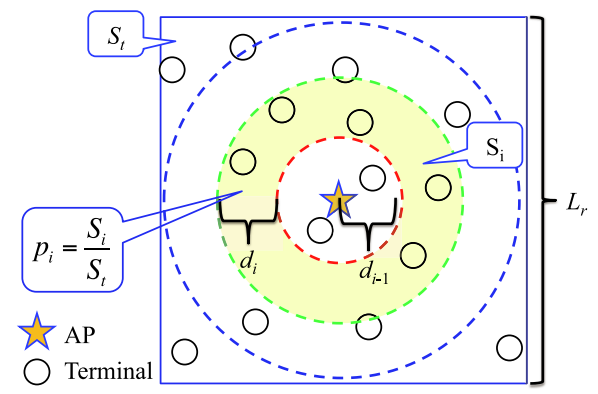

Fig. 4 Throughput prediction based on area ratio.

the area ratio of the system field wherein the terminal exists. This method uses a circular area whose radius is defined by the transmission rate. In this method, network structure is limited to a uniform distribution. For simplicity, this study assumes that the AP is located at the center of the system field (the origin of the field) and the system field is a square with sides $L_{r}$. In this method, $S_{t}\left(=L_{r}^{2}\right)$ denotes the area of the field. $S_{i}$ is the area size beyond radius $d_{i-1}\left(d_{i}\right.$ is the threshold of the distance at which the transmission rate changes. $\left.d_{0}=0\right)$ ) but within radius $d_{i}\left(>d_{i-1}\right)$. In Fig. 4 , this area means that the terminal exists in the colored area. $S_{i}$ can be obtained by Eq. (10):

$$
S_{i}=\pi\left(d_{i}^{2}-d_{i-1}^{2}\right) \quad(i=1,2, \cdots, 8) .
$$

Thus, the ratio of the number of terminals $p_{i}$ whose transmission rate is $b_{i}$ is described by Eq. (11):

$$
p_{i}=S_{i}\left(S_{t}\right)^{-1} \quad(i=1,2, \cdots, 8) .
$$

By substituting $p_{i}$ for Eq. (5), the proposed method can predict the throughput. In addition, this method can be performed in $O(1)$ time.

\subsection{Algorithm of the Proposed Method}

This section explains the algorithm of the proposed method. From the above description, the proposed method uses a target problem for throughput prediction if the terminals are placed according to a two-dimensional normal distribution. If the target problem is used for the prediction, the input of the proposed method is $\sigma$. Conversely, the proposed method uses the area ratio for throughput prediction if the

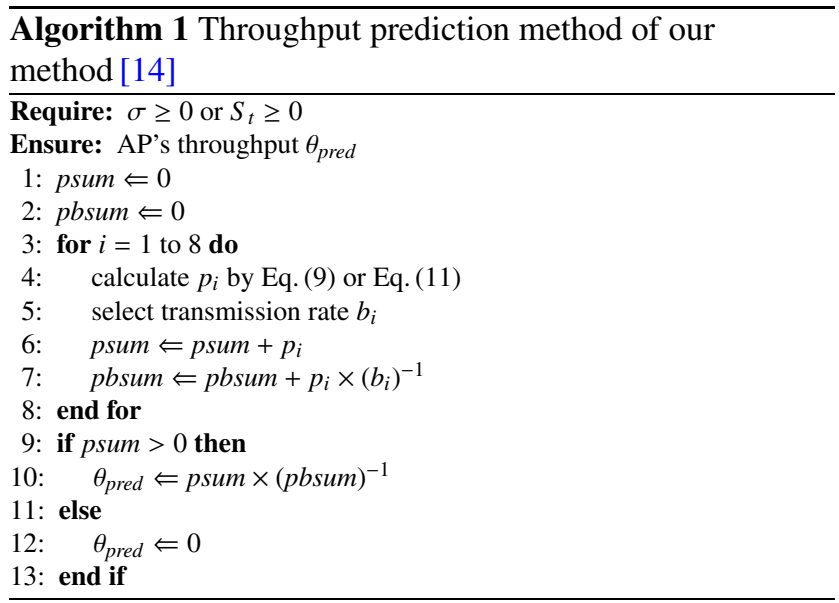

APs are placed according to uniform distribution. In this situation, the input of the proposed method is $S_{t}$. An estimation method for $\sigma$ has been proposed previously [21]. Moreover, this study assumes that $S_{t}$ can be measured in advance. The output is the prediction throughput. Based on the above, Algorithm 1 summarizes the procedure of the throughput prediction method [14].

\section{Derivation of Effective Transmission Rate Consider- ing CSMA/CA and Frame Collision}

This section explains the derivation of an effective transmission rate considering frame collision percentage and CSMA/CA access control.

\subsection{Transmission Rate Considering CSMA/CA}

Here we discuss the transmission rate considering media access control by CSMA/CA. First, we explain the transmission rate of a UDP flow case. In CSMA/CA, the time required to transmit one data frame $T_{\text {Total_UDP }}$ is obtained by Eq. (12):

$$
T_{\text {Total_UDP }}=T_{\text {DIFS }}+T_{\text {Back }}+T_{U D P-D a t a}+T_{S I F S}+T_{\text {Ack }},
$$

where $T_{D I F S}$ and $T_{S I F S}$ are the DIFS time and SIFS time, respectively. $T_{B a c k}$ denotes the back-off time. In addition, $T_{U D P-D a t a}$ and $T_{A c k}$ represent the transfer time of a data frame and an acknowledgement frame, respectively. Note that this paper assumes that IEEE802.11g (PHY) [22] is used for the WLAN environment. In IEEE802.11g, $T_{D I F S}$ and $T_{\text {SIFS }}$ are $34 \mu \mathrm{s}$ and $16 \mu \mathrm{s}$, respectively. To calculate the theoretical performance of the throughput, the back-off time can be obtained by Eq. (13) [23]:

$$
\text { back-off }=0.5 C W_{\min } \times \text { SlotTime, }
$$

where $C W_{\min }$ (minimum of Contention Window) and SlotTime denote the minimum contention window and the slot time interval specified by IEEE802.11, respectively. In IEEE $802.11 \mathrm{~g}$, note that SlotTime is $9 \mu \mathrm{s}$. Therefore, $T_{B a c k}$ is 
$67.5 \mu \mathrm{s}$. Furthermore, $T_{U D P-D a t a}$ and $T_{A c k}$ depend on the data frame size $F S_{U D P-D a t a}$, acknowledgement frame size $F S_{A c k}$, and transmission rate. $F S_{U D P-D a t a}$ differs for each terminal; however, $F S_{A c k}$ is always 224 bits. The theoretical value of the transmission rate is shown in Table 1 . The effective transmission rate of UDP communication can be obtained by $F S_{U D P-D a t a} / T_{\text {Total_UDP }}$. This effective transmission rate does not consider frame collision.

Next, we explain the transmission rate of a TCP flow case. In this paper, we model the behavior of TCP as follows. In TCP communication, let $v$ be the number of data segments acknowledged by a received TCP-Ack. Many TCP receiver implementations send one cumulative ACK for some consecutive packets received (e.g., delayed ACK [24], [25]). In this condition, the total time required to transmit $v$ data frames $T_{\text {Total_TCP }}$ can be calculated by Eq. (14):

$$
\begin{aligned}
T_{\text {Total_TCP }}= & v\left(T_{\text {DIFS }}+T_{\text {Back }}+T_{T C P-D a t a}+T_{\text {SIFS }}+T_{A c k}\right) \\
& +T_{D I F S}+T_{\text {Back }}+T_{T C P-A c k}+T_{S I F S}+T_{A c k},
\end{aligned}
$$

where $T_{T C P-D a t a}$ and $T_{T C P-A c k}$ are the transfer time of TCPData and TCP-Ack, respectively. The mean of the other variables $\left(T_{D I F S}, T_{B a c k}, T_{S I F S}\right.$, and $\left.T_{A c k}\right)$ are the same as the UDP case. Here the effective transmission rate of TCP communication can be obtained by $v\left(F S_{\text {TCP-Data }} / T_{\text {Total_TCP }}\right)$ where $F S_{T C P-D a t a}$ is the data frame size of TCP-Data. It is expected that the effective transmission rate will be affected by the value of $v$. A detailed evaluation that considers $v$ is planned for future work.

\subsection{Probability of Frame Collision Corresponding to the Number of Terminals}

Here we discuss the probability of a frame collision corresponding to the number of terminals with reference to previous studies [26], [27]. [26], [27] are called Bianchi's model. In Bianchi's model, wireless channels have two usage states (see Fig. 5), busy (transmission on the channel) and idle (channel not used for transmission). Let $\xi_{i}$ and $\xi_{b}$ be the probabilities that a terminal accesses the channel after an idle state and a busy state, respectively. Let $a$ represent the probability that the channel becomes busy given that it was idle in the previous slot. Similarly, let $b$ be the probability that the channel becomes idle given that it was busy in the previous slot. $a$ and $b$ can be obtained from Eqs. (15) and (16), respectively:

$$
a=1-\left(1-\xi_{i}\right)^{n},
$$

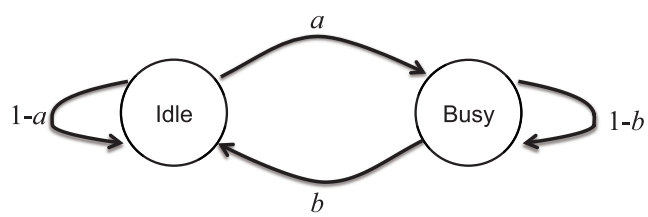

Fig.5 Wireless channel state of Bianchi's model.

$$
b=\left(1-\xi_{b}\right)^{n},
$$

where $n$ is the number of wireless terminals using the same channel. Here $P_{i}$ denotes the probability that a channel is idle. Using $P_{i}$, the collision percentage of a frame transmitted by a wireless terminal can be obtained by Eq. (17):

$$
p_{c o l}=P_{i}\left[1-\left(1-\xi_{i}\right)^{n-1}\right]+\left(1-P_{i}\right)\left[1-\left(1-\xi_{b}\right)^{n-1}\right] .
$$

When the channel access probability of a terminal is $\xi$ and the channel access probability is the same regardless of the channel status, $\xi_{i}=\xi_{b}=\xi$. As a result, $\xi$ can be obtained using the average contention window size $C W_{\text {avg }}$ in Eq. (18):

$$
\xi=P_{i} \xi_{i}+\left(1-P_{i}\right) \xi_{b}=C W_{\text {avg }}^{-1} .
$$

Using Eq. (18), Eq. (17) can be rewritten as follows:

$$
p_{c o l}=1-\left(1-C W_{\text {avg }}^{-1}\right)^{n-1} .
$$

From Eq. (19), the successful transmission probability of a frame is $1-p_{c o l}$. The effective transmission rate considering data frame collision can be obtained [28] by multiplying this value and the effective transmission rate obtained in Sect. 4.1.

\section{Evaluation}

This section explains the simulation environment and compares the prediction value $\left(\theta_{\text {pred }}\right)$ calculated by the proposed method or the existing method [9] using the effective transmission rate (Sect. 4) with a simulation value $\left(\theta_{n s 2}\right)$ obtained using NS2 simulation [10]. The goal of this paper is to improve our previously proposed method [14] with respect to both media access control and frame collision. Therefore, the result of the existing method is shown for reference. In addition, the absolute error and relative error between the simulation value and the prediction value are evaluated. Here the terminal distribution follows two-dimensional normal distribution and uniform distribution.

\subsection{Simulation Environments}

This evaluation assumes a two-dimensional plane. The AP is placed at $(0,0)$, and the terminals are distributed according to two-dimensional $N\left(0, \sigma^{2}\right)$ or uniform distribution. The number of terminals is 10 or 20 . In this evaluation, the range of uniform distribution is $\left[-L_{r} / 2, L_{r} / 2\right]$. Note that, in practice, the recommended number of terminals that can connect to a single consumer AP is approximately 20 [29]. [29] indicates the maximum connection number is 25 . As a result, we adopt the number 20 because there is almost no difference between 25 and 20. Figure 6 shows the network model. The goal of this evaluation is to compare the difference between the prediction value and the simulation value. Therefore, in the evaluation, the AP knows the number of terminals $N$, data frame size $F S_{U D P-D a t a}$ or $F S_{T C P-D a t a}$, and $\sigma /$ the field size $S_{t}\left(=L_{r}^{2}\right)$. Various studies 
Table 2 Relationship between distance and effective transmission rate (UDP).

\begin{tabular}{|l|l|l|l|l|l|l|l|l|}
\hline Index $i$ & 1 & 2 & 3 & 4 & 5 & 6 & 7 & 8 \\
\hline Threshold of distance $d_{i}$ from AP m & 5 & 7 & 9 & 20 & 25 & 40 & 50 & 60 \\
\hline Effective transmission rate $b e_{i}$ Mbps & 28.9 & 27.0 & 22.5 & 16.8 & 13.4 & 9.6 & 7.5 & 5.2 \\
\hline
\end{tabular}

Table 3 Relationship between distance and effective transmission rate (TCP)

\begin{tabular}{|l|l|l|l|l|l|l|l|l|}
\hline Index $i$ & 1 & 2 & 3 & 4 & 5 & 6 & 7 & 8 \\
\hline Threshold of distance $d_{i}$ from AP m & 5 & 7 & 9 & 20 & 25 & 40 & 50 & 60 \\
\hline Effective transmission rate $b e_{i}$ Mbps & 29.2 & 27.3 & 22.6 & 16.9 & 13.5 & 9.6 & 7.5 & 5.2 \\
\hline
\end{tabular}

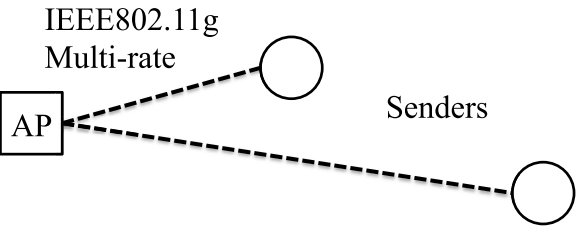

Fig. 6 Network model.

have investigated the flow analysis of Internet traffic [30][32]. The frame size can be estimated using this knowledge. In future, we will evaluate the proposed method considering the frame size estimation method. In this evaluation, the terminals use IEEE802.11g [22] and do not move. Note that a previous study has proposed an estimation method for terminal distribution [14]. Therefore, the proposed method can be used if terminals move. If terminals move in the network, error increases by some effect such as fading attenuation and errors in the estimation of terminal distribution. However, this evaluation's motivation is to compare the throughput obtained by NS2 and the proposed method. Therefore, we will evaluate the proposed method considering terminal movement in future. UDP and TCP are used as the transport protocols, and the Constant Bit Rate (CBR) (30 Mbps) and the File Transfer Protocol (FTP) are the applications for each transport protocol, respectively. The data frame size $F S_{U D P-D a t a}$ and $F S_{T C P-D a t a}$ are 1058 Bytes and 1098 Bytes, respectively. In this evaluation, we consider a case in which wireless terminals are the senders and generate $180 \mathrm{~s}$ of traffic (each terminal generates a single flow). Note that we have confirmed the congestion window size is stable for about 30 seconds even when many low-rate flows are included. Therefore, 180 seconds is long enough for the simulation. Future work includes an evaluation that will consider bidirectional flows. The prediction value is calculated using the effective stepped transmission rate (Table 2 shows the UDP case and Table 3 shows the TCP case), and in the NS2 simulation, each terminal and the AP use the theoretical stepped transmission rate (Table 1). In the TCP case, the $v$ value is one-half of the receiving window size (64 packets). As mentioned above, more complex transmission rate selection algorithms are installed for the real machines. However, the detail of the algorithm is closed for the public. Therefore, we use the simple transmission rate selection algorithm for evaluations. In the future, we will evaluate our proposal considering more realistic transmission rate selection algorithms. Table 4 summarizes other
Table 4 Simulation parameters.

\begin{tabular}{|l|l|}
\hline Simulator & ns-2 (ver.2.34) \\
\hline WLAN & IEEE802.11g (Multi-rate) \\
\hline Radio wave propagation & Two ray ground \\
\hline AP's buffer size & 250 packets \\
\hline Transport protocol & UDP, TCP-Reno with Sack \\
\hline Segment size & 1000 byte \\
\hline Simulation time & 180 sec \\
\hline Receiving window size & 128 packet \\
\hline
\end{tabular}

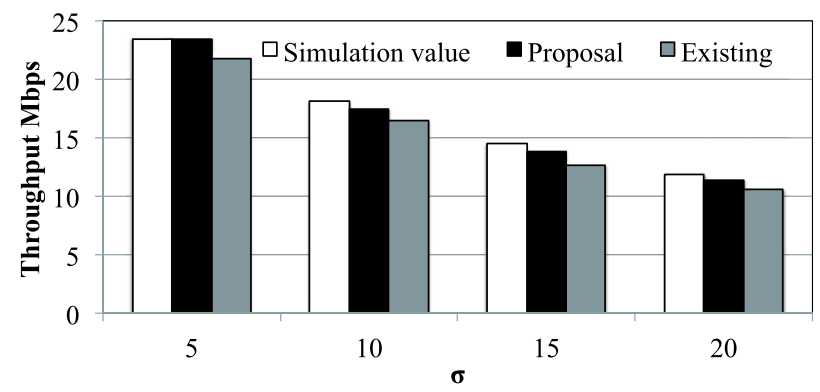

Fig. 7 Comparison of simulation value and prediction value (UDP; normal distribution; number of terminals, 10).

simulation parameters. The simulation results are the averages of 40 trials for each $\sigma$ or $L_{r}$. In addition, if the distance between the AP and the terminal is greater than $60 \mathrm{~m}$, the terminal's transmission rate is 0 bps (i.e., the terminal cannot communicate with the AP). The relationship between $\sigma$ and the distance $D_{\max }$ between the AP and the terminal farthest from the AP is obtained by Eq. (20) [21]:

$$
D_{\max }=4 \sigma .
$$

Therefore, the side length of the system field $L_{r}$ is $8 \sigma$ for all values of $\sigma$. Here we determine the absolute error $E_{a}$ and relative error $E_{r}$ in throughput estimates between the prediction value $\theta_{\text {pred }}$ and the simulation value $\theta_{n s 2}$ using Eqs. (21) and (22):

$$
\begin{aligned}
& E_{a}=\left|\theta_{\text {pred }}-\theta_{n s 2}\right|, \\
& E_{r}=E_{a}\left(\theta_{n s 2}\right)^{-1} .
\end{aligned}
$$

\subsection{Results}

This section shows the result for each transport protocol. 
Table 5 Error between simulation value and prediction value for each $\sigma$ with UDP.

\begin{tabular}{|c|c|c|c|c|c|c|c|c|}
\hline \multirow{3}{*}{$\sigma$} & \multicolumn{4}{|c|}{$N: 10$} & \multicolumn{4}{c|}{$N: 20$} \\
\cline { 2 - 9 } & \multicolumn{2}{|c|}{ Proposal } & \multicolumn{2}{|c|}{ Existing } & \multicolumn{3}{c|}{ Proposal } & \multicolumn{2}{c|}{ Existing } \\
\cline { 2 - 9 } & $E_{a}$ Mbps & $E_{r} \%$ & $E_{a}$ Mbps & $E_{r} \%$ & $E_{a}$ Mbps & $E_{r} \%$ & $E_{a}$ Mbps & $E_{r} \%$ \\
\hline 5 & 0.08 & 0.38 & 1.70 & 7.28 & 1.37 & 6.41 & 0.25 & 1.15 \\
\hline 10 & 0.61 & 3.36 & 1.62 & 8.92 & 0.73 & 4.48 & 0.01 & 0.08 \\
\hline 15 & 0.65 & 4.48 & 1.79 & 12.43 & 0.18 & 1.41 & 0.59 & 4.42 \\
\hline 20 & 0.51 & 4.31 & 1.32 & 11.12 & 0.28 & 2.61 & 0.44 & 4.03 \\
\hline
\end{tabular}

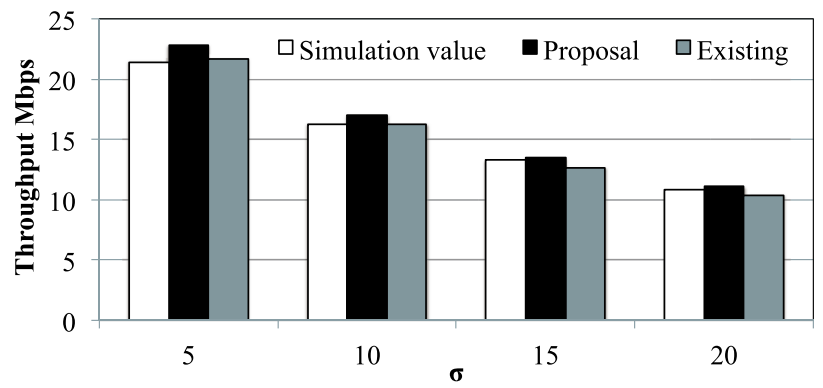

Fig. 8 Comparison of simulation value and prediction value (UDP; normal distribution; number of terminals, 20).

\subsubsection{UDP Case}

First, we discuss the results when the terminals were distributed according to two-dimensional $N\left(0, \sigma^{2}\right)$. Figures 7 and 8 show comparisons of the simulation value and the prediction value when the number of terminals was 10 and 20 , respectively. In Figs. 7 and 8, the horizontal axis plots $\sigma$, and the vertical axis plots throughput. These simulation results show that the proposed method and the existing method demonstrate nearly the same trend as the simulation value. Table 5 summarizes $E_{a}$ and $E_{r}$ for each $N$ and $\sigma$. From Table 5, when $N$ is 10 and the proposed method is used for prediction, $E_{a}$ and $E_{r}$ are less than $0.7 \mathrm{Mbps}$ and $5 \%$ for each $\sigma$, respectively. When $N$ is 20 , both $E_{a}$ and $E_{r}$ are small if $\sigma=\{10,15,20\}$. In other words, if the terminals are distributed widely, the errors are small. Conversely, when $\sigma$ is $5, E_{a}$ and $E_{r}$ are $1.37 \mathrm{Mbps}$ and $6.41 \%$, respectively. Therefore, if terminal density is high, both $E_{a}$ and $E_{r}$ are large. Furthermore, from Fig. $8, \theta_{\text {pred }}$ is larger than $\theta_{n s 2}$ for each $\sigma$ because each terminal sends data packets using CBR at $30 \mathrm{Mbps}$. Moreover, the number of terminals is large; therefore, contention occurs frequently. As a result, buffer overflow occurs in the terminals. Note that this paper does not focus on buffer overflow (Sect. 4). For that reason, errors increase. In addition, when $N$ is $10, E_{a}$ and $E_{r}$ of the existing method are less than $1.8 \mathrm{Mbps}$ and $12.5 \%$, respectively. Furthermore, when $N$ is $20, E_{a}$ and $E_{r}$ of the existing method are less than $0.6 \mathrm{Mbps}$ and $4.5 \%$, respectively. From this result, if the number of terminals is small, the prediction accuracy of the proposed method is higher than that of the existing method. Moreover, if the number of terminals is large and $\sigma$ is small, the prediction accuracy of the existing method is higher than that of the proposed method.

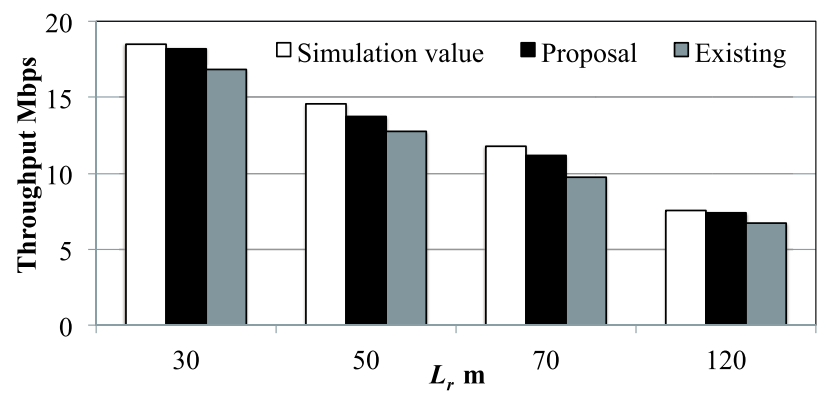

Fig. 9 Comparison of simulation value and prediction value (UDP; uniform distribution; number of terminals, 10).

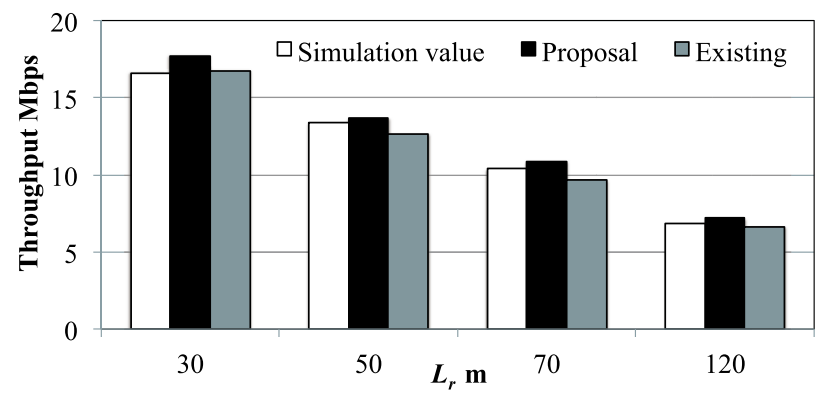

Fig. 10 Comparison of simulation value and prediction value (UDP; uniform distribution; number of terminals, 20).

Next, we discuss the results when terminals were distributed uniformly. Figures 9 and 10 plot comparisons of the simulation value and the prediction value when the number of terminals was 10 and 20, respectively. In Figs. 9 and 10, the horizontal axis is the side length of the system field $L_{r}$, and the vertical axis is throughput. These simulations show that the proposed method demonstrates nearly the same trend as the simulation value. Table 6 summarizes $E_{a}$ and $E_{r}$ for each $N$ and $L_{r}$. From Table 6 , when $N$ is 10, $E_{a}$ and $E_{r}$ are less than $0.9 \mathrm{Mbps}$ and $6 \%$ for each $L_{r}$, respectively. When $N$ is 20 , both $E_{a}$ and $E_{r}$ are small if $L_{r}=\{50,70,120\} \mathrm{m}$. Conversely, when $L_{r}$ is $30, E_{a}$ and $E_{r}$ are $1.11 \mathrm{Mbps}$ and $6.72 \%$, respectively. Therefore, if terminal density is high, both $E_{a}$ and $E_{r}$ are large, which is consistent with the previous evaluation. The reason is similar to the previous evaluation. Note that, when $N$ is $10, E_{a}$ and $E_{r}$ of the existing method are less than $2.1 \mathrm{Mbps}$ and $17.5 \%$, respectively. Furthermore, when $N$ is $20, E_{a}$ and $E_{r}$ of the existing method are less than $0.72 \mathrm{Mbps}$ and $6.9 \%$, respectively. Thus, if the number of terminals is small, the prediction accuracy of the proposed method is higher than 
Table 6 Error between simulation and prediction values for each $L_{r}$ with UDP.

\begin{tabular}{|c|c|c|c|c|c|c|c|c|}
\hline \multirow{3}{*}{$L_{r} \mathrm{~m}$} & \multicolumn{4}{|c|}{$N: 10$} & \multicolumn{4}{c|}{$N: 20$} \\
\cline { 2 - 9 } & \multicolumn{2}{|c|}{ Proposal } & \multicolumn{2}{|c|}{ Existing } & \multicolumn{3}{c|}{ Proposal } & \multicolumn{2}{c|}{ Existing } \\
\cline { 2 - 9 } & $E_{a}$ Mbps & $E_{r} \%$ & $E_{a}$ Mbps & $E_{r} \%$ & $E_{a}$ Mbps & $E_{r} \%$ & $E_{a}$ Mbps & $E_{r} \%$ \\
\hline 30 & 0.27 & 1.47 & 1.65 & 8.93 & 1.11 & 6.72 & 0.15 & 0.90 \\
\hline 50 & 0.82 & 5.65 & 1.81 & 12.47 & 0.31 & 2.31 & 0.69 & 5.13 \\
\hline 70 & 0.62 & 5.29 & 2.05 & 17.35 & 0.50 & 4.84 & 0.71 & 6.81 \\
\hline 120 & 0.15 & 1.95 & 0.83 & 11.01 & 0.34 & 4.90 & 0.22 & 3.20 \\
\hline
\end{tabular}

Table 7 Collision percentage for each $\sigma$ in NS2 with UDP.

\begin{tabular}{|c|c|c|}
\hline$\sigma$ & $N: 10$ & $N: 20$ \\
\hline 5 & $31.38 \%$ & $42.17 \%$ \\
\hline 10 & $31.38 \%$ & $42.14 \%$ \\
\hline 15 & $31.36 \%$ & $42.13 \%$ \\
\hline 20 & $31.39 \%$ & $42.16 \%$ \\
\hline
\end{tabular}

Table 8 Collision percentage for each $L_{r}$ in NS2 with UDP.

\begin{tabular}{|c|c|c|}
\hline$L_{r}$ & $N: 10$ & $N: 20$ \\
\hline 30 & $31.37 \%$ & $42.14 \%$ \\
\hline 50 & $31.38 \%$ & $42.14 \%$ \\
\hline 70 & $31.37 \%$ & $42.17 \%$ \\
\hline 120 & $31.37 \%$ & $42.18 \%$ \\
\hline
\end{tabular}

that of the existing method.

Finally, Tables 7 and 8 show the collision percentage for each $\sigma$ in the simulation. The collision percentage in the simulation $P_{\text {colnss } 2}$ can be calculated as follows:

$$
P_{\text {col_ns } 2}=N_{\text {col }} N_{\text {send }}^{-1} \text {. }
$$

In Eq. (23), $N_{c o l}$ and $N_{\text {send }}$ denote the number of total collision frames and total sending frames in all terminals, respectively. From Tables 7 and 8, the collision percentage does not depend on the distribution of terminal. As indicated in Sect. 4.2, collision percentage depends on the number of terminals.

From the simulation results, if the number of terminals is small and terminals are distributed widely, $E_{a}$ and $E_{r}$ are very small. However, if the number of terminals is large and terminal density is high, errors increase. This issue can be resolved by considering buffer overflow, which will be addressed in future work.

\subsubsection{TCP Case}

Here we show the results of the case when terminals were distributed according to two-dimensional $N\left(0, \sigma^{2}\right)$. Figures 11 and 12 plot comparisons of the simulation value and the prediction value when the number of terminals was 10 and 20, respectively. In Figs. 11 and 12, the horizontal axis plots $\sigma$, and the vertical axis plots throughput. These simulation results show that the proposed method demonstrates nearly the same trend as the simulation value, similar to the UDP case. Table 9 shows $E_{a}$ and $E_{r}$ for each $N$ and $\sigma$. From Table 9, when $N$ is 10, the maximum value of both $E_{a}$ and $E_{r}$ are approximately $2 \mathrm{Mbps}$ and $15 \%$, respectively. When $N$ is 20 , the maximum values of both $E_{a}$

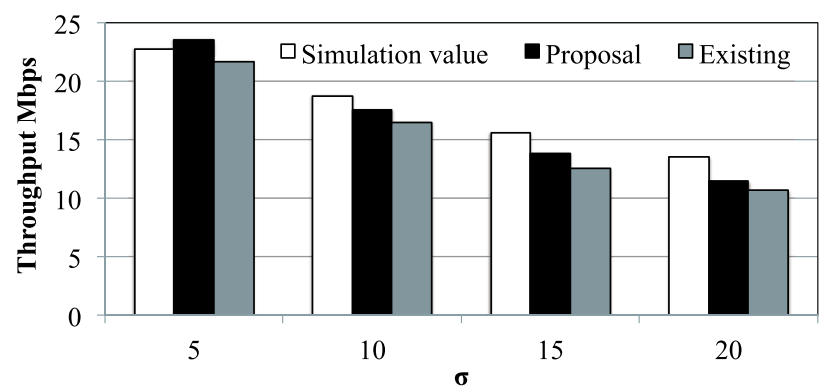

Fig. 11 Comparison of simulation value and prediction value (TCP; normal distribution; number of terminals, 10).

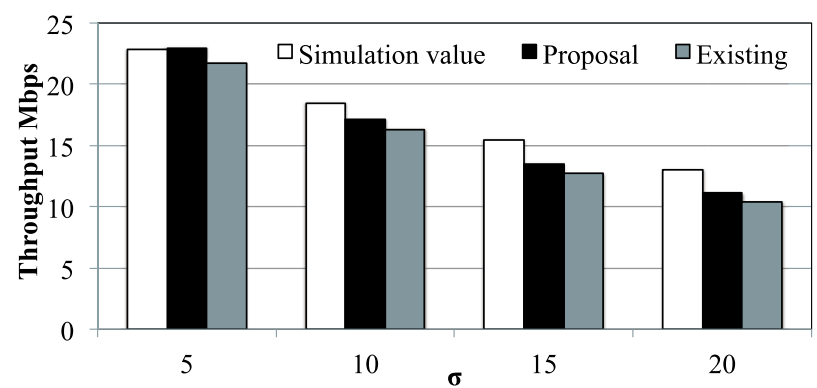

Fig. 12 Comparison of simulation value and prediction value (TCP; normal distribution; number of terminals, 20).

and $E_{r}$ are approximately $1.9 \mathrm{Mbps}$ and $14 \%$, respectively. Therefore, if TCP is used as the transport protocol, both $E_{a}$ and $E_{r}$ are large compared to UDP. Furthermore, if the value of $\sigma$ is $5, \theta_{n s 2}$ is less than $\theta_{\text {pred }}$ because buffer overflow occurs in the terminals, similar to the UDP case. Conversely, if $\sigma=\{10,15,20\}, \theta_{n s 2}$ is greater than $\theta_{\text {pred }}$, because TCP communicates on the basis of window flow control and congestion control. However, these control are not reflected in the transmission rate of the TCP case (Table 3) completely. In the case of TCP unlike UDP, estimated throughput of the proposed method is influenced significantly by the error of the calculated effective transmission rate of TCP. Moreover, the increased window size is not ideal due to the loss of TCP-Data and TCP-Ack segments. For that reason, error increases. We think that the correct adjustment of the parameter $v$ related with the effective transmission rate of TCP can improve the estimate precision. In addition, when $N$ is $10, E_{a}$ and $E_{r}$ of the existing method are less than 3.1 Mbps and $21.2 \%$, respectively. Furthermore, when $N$ is 20 , the maximum $E_{a}$ and $E_{r}$ of the existing method are $2.75 \mathrm{Mbps}$ and $20.0 \%$, respectively. 
Table 9 Error between simulation value and prediction value for each $\sigma$ with TCP.

\begin{tabular}{|c|c|c|c|c|c|c|c|c|}
\hline \multirow{3}{*}{$\sigma$} & \multicolumn{4}{|c|}{$N: 10$} & \multicolumn{4}{c|}{$N: 20$} \\
\cline { 2 - 9 } & \multicolumn{2}{|c|}{ Proposal } & \multicolumn{2}{|c|}{ Existing } & \multicolumn{2}{c|}{ Proposal } & \multicolumn{2}{c|}{ Existing } \\
\cline { 2 - 9 } & $E_{a}$ Mbps & $E_{r} \%$ & $E_{a}$ Mbps & $E_{r} \%$ & $E_{a}$ Mbps & $E_{r} \%$ & $E_{a}$ Mbps & $E_{r} \%$ \\
\hline 5 & 0.77 & 3.39 & 1.13 & 4.97 & 0.11 & 0.51 & 1.11 & 4.86 \\
\hline 10 & 1.19 & 6.38 & 2.26 & 12.06 & 1.34 & 7.26 & 2.14 & 11.58 \\
\hline 15 & 1.73 & 11.09 & 3.04 & 19.50 & 1.96 & 12.69 & 2.75 & 17.79 \\
\hline 20 & 2.07 & 15.33 & 2.86 & 21.18 & 1.90 & 14.61 & 2.61 & 20.00 \\
\hline
\end{tabular}

Table 10 Error between simulation value and prediction value for each $L_{r}$ with TCP.

\begin{tabular}{|c|c|c|c|c|c|c|c|c|}
\hline \multirow{3}{*}{$L_{r} \mathrm{~m}$} & \multicolumn{4}{|c|}{$N: 10$} & \multicolumn{4}{c|}{$N: 20$} \\
\cline { 2 - 9 } & \multicolumn{2}{|c|}{ Proposal } & \multicolumn{2}{|c|}{ Existing } & \multicolumn{3}{c|}{ Proposal } & \multicolumn{2}{c|}{ Existing } \\
\cline { 2 - 9 } & $E_{a}$ Mbps & $E_{r} \%$ & $E_{a}$ Mbps & $E_{r} \%$ & $E_{a}$ Mbps & $E_{r} \%$ & $E_{a}$ Mbps & $E_{r} \%$ \\
\hline 30 & 0.06 & 0.59 & 1.58 & 8.61 & 0.59 & 3.19 & 1.69 & 9.18 \\
\hline 50 & 2.11 & 13.26 & 3.21 & 20.20 & 1.86 & 12.13 & 2.63 & 17.19 \\
\hline 70 & 0.84 & 6.94 & 2.28 & 18.85 & 1.42 & 11.55 & 2.72 & 21.96 \\
\hline 120 & 0.83 & 10.00 & 1.53 & 18.52 & 0.98 & 12.98 & 1.55 & 18.82 \\
\hline
\end{tabular}

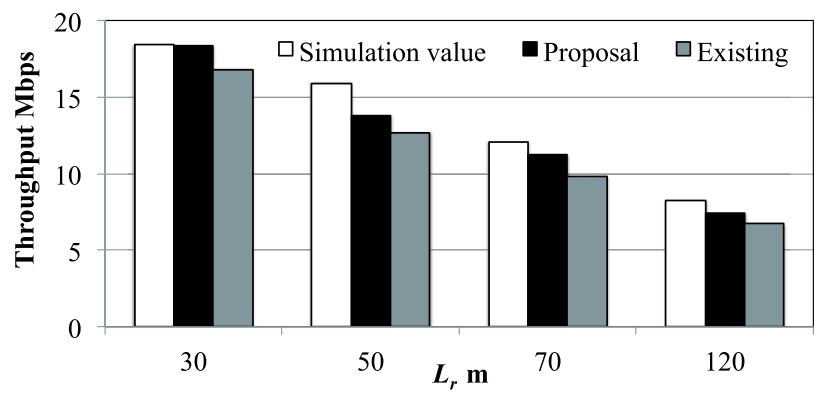

Fig. 13 Comparison of simulation value and prediction value (TCP; uniform distribution; number of terminals, 10).

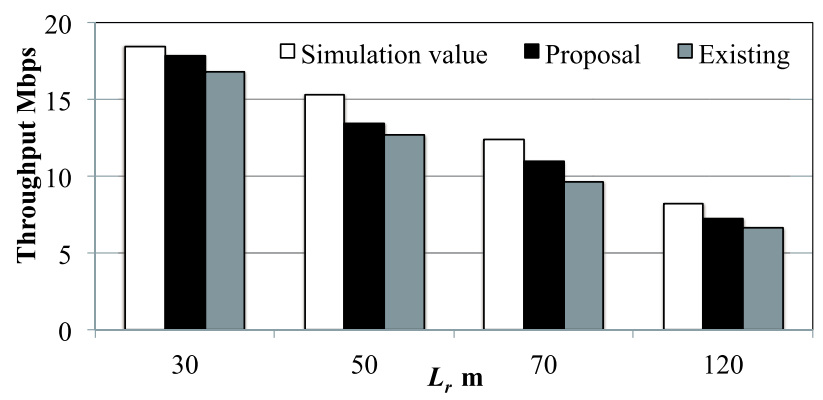

Fig. 14 Comparison of simulation value and prediction value (TCP; uniform distribution; number of terminals, 20).

Here we explain the results of the case when the terminals were distributed uniformly. Figures 13 and 14 plot comparisons of the simulation value and the prediction value when the number of terminals was 10 and 20, respectively. In Figs. 13 and 14, the horizontal axis is the side length of the system field $L_{r}$, and the vertical axis is throughput. These simulations show that the proposed method demonstrates nearly the same trend as the simulation value, similar to the UDP case. Table 10 summarizes $E_{a}$ and $E_{r}$ for each $N$ and $L_{r}$. From Table 10, when $N$ is 10 , the maximum value of $E_{a}$ and $E_{r}$ are approximately $2.1 \mathrm{Mbps}$ and $13 \%$ for each
Table 11 Collision percentage for each $\sigma$ in NS2 with TCP

\begin{tabular}{|c|c|c|}
\hline$\sigma$ & $N: 10$ & $N: 20$ \\
\hline 5 & $13.54 \%$ & $15.67 \%$ \\
\hline 10 & $13.80 \%$ & $15.82 \%$ \\
\hline 15 & $13.79 \%$ & $16.08 \%$ \\
\hline 20 & $13.82 \%$ & $15.93 \%$ \\
\hline
\end{tabular}

Table 12 Collision percentage for each $L_{r}$ in NS2 with TCP.

\begin{tabular}{|c|c|c|}
\hline$L_{r}$ & $N: 10$ & $N: 20$ \\
\hline 30 & $13.73 \%$ & $15.81 \%$ \\
\hline 50 & $13.93 \%$ & $16.01 \%$ \\
\hline 70 & $14.13 \%$ & $16.52 \%$ \\
\hline 120 & $14.75 \%$ & $17.21 \%$ \\
\hline
\end{tabular}

$L_{r}$, respectively. Conversely, when $N$ is 20 , the maximum values of $E_{a}$ and $E_{r}$ are approximately $1.8 \mathrm{Mbps}$ and $12 \%$, respectively. The reason for this trend in error is similar to the previous evaluation. Moreover, when $N$ is 10 , the maximum $E_{a}$ and $E_{r}$ of the existing method are $3.21 \mathrm{Mbps}$ and $20.2 \%$, respectively. Furthermore, when $N$ is 20 , the maximum $E_{a}$ and $E_{r}$ of the existing method are $2.72 \mathrm{Mbps}$ and $21.96 \%$, respectively.

Finally, Tables 11 and 12 show the collision percentage for each $\sigma$ in the simulation. Note that the collision percentage in the simulation $P_{\text {col_ns2 }}$ can be calculated by Eq. (23) (the same as the UDP case). Note that we only show the collision percentage for TCP-Data. From Tables 11 and 12, the collision percentage does not depend heavily on the distribution of terminals. Furthermore, the probability value is less than that of the UDP case (Tables 7 and 8) because the transmission rate in the transport layer decreases due to TCP congestion control. As a result, the number of collisions decreases compared to the UDP case. In addition, as indicated in Sect. 4.2, collision percentage depends on the number of terminals (same as the UDP case).

From the simulation results, if TCP is used for the transport protocol, $E_{a}$ and $E_{r}$ are greater than that of UDP. 
This issue can be resolved by considering a more realistic transmission rate model, which will be addressed in future work.

\section{Conclusion}

Our previous study [14] proposed and evaluated throughput prediction methods that consider the terminal distribution of APs in multi-rate WLAN environments. However, these evaluations did not compare the predicted throughput value to a simulation value in consideration of CSMA/CA and frame collision. In this study, we improved the throughput prediction formula to consider data frame collision and CSMA/CA. Then, we compared the prediction value and the simulation value obtained by the NS2 network simulator in a multi-rate environment. The results show that the maximum relative error of the proposed method is approximately $6 \%$ and $15 \%$ for UDP and TCP, respectively, while that is approximately $17 \%$ and $21 \%$ in existing method. Future work will include the following evaluations.

- Improvement of the prediction method considering buffer overflow, existence of obstacles and the strength of received signals, etc.

- Evaluation considering terminal movement, bidirectional flow and other terminal distribution

- Evaluation by using more complex rate selection algorithms such as ARF (Auto Rate Fallback) [33], [34]

- Modeling of more realistic TCP control

- Modeling of a hidden node problem and an exposed node problem

- Application to network design (e.g., AP selection and AP placement problems)

\section{Acknowledgments}

This work was partly supported by the Japan Society for the Promotion of Science KAKENHI Grant Numbers 26420367, 26280032, 15K00431, and 15H02688, and by the Project Research Grants of the Graduate School of Information Sciences, Hiroshima City University.

\section{References}

[1] R. Hamamoto, C. Takano, H. Obata, and K. Ishida, "Improvement of throughput prediction method for access point in multi-rate WLANs considering media access control and frame collision," Proc. 8th International Workshop on ASON 2015, pp.227-233, 2015.

[2] IEEE Standard, "Wireless LAN medium access control (MAC) and physical layer (PHY) specifications," ANSI/IEEE Std 802.11, 2012.

[3] A. Balachandran, P. Bahl, and G.M. Voelker, "Hot-Spot congestion relief in public-area wireless networks," Proc. IEEE WMCSA 2002, pp.70-82, 2002.

[4] M.F. Tuysuz and H.A. Mantar, "Access point selection for improving the voice quality and overall throughput in wireless LANs," Proc. IEEE SoftCOM 2010, pp.165-169, 2010.

[5] Y. Lin, W. Yu, and Y. Lostanlen, "Optimization of wireless access point placement in realistic urban heterogeneous networks," Proc. IEEE GLOBECOM 2012, pp.4963-4968, 2012.
[6] Y. Imagaki, K. Kashiki, K. Yamazaki, and A. Yamaguchi, "Novel Wi-Fi throughput estimation method considering CSMA/CA behavior," Proc. IEEE 75th VTC Spring, pp.1-5, 2012.

[7] C. Rattaro and P. Belzarena, "Throughput prediction in wireless networks using statistical learning," Proc. Latin-American Workshop on Dynamic Networks, p.4, 2010.

[8] I. Tinnirello and G. Bianchi, "Rethinking the IEEE 802.11e EDCA performance modeling methodology," IEEE/ACM Trans. Netw., vol.18, no.2, pp.540-553, 2010.

[9] K. Medepalli and F.A. Tobagi, "Throughput analysis of IEEE 802.11 wireless LANs using an average cycle time approach," Proc. IEEE GLOBECOM 2005, pp.3007-3011, 2005.

[10] The Network Simulator ns-2, Project web page available at http://www.isi.edu/nsnam/ns/, 2015.

[11] F. Miki, D. Nobayashi, Y. Fukuda, and T. Ikenaga, "Performance evaluation of multirate communication in wireless LANs," Proc. 7th IEEE CCNC 2010, pp.1-3, 2010.

[12] S. Miyata, T. Murase, and K. Yamaoka, "Novel access-point selection for user QoS and system optimization based on user cooperative moving," IEICE Trans. Commun., vol.E95-B, no.6, pp.1953-1964, June 2012.

[13] R. Hamamoto, C. Takano, H. Obata, K. Ishida, and T. Murase, "An access point selection mechanism based on cooperation of access points and users movement," Proc. IFIP/IEEE IM 2015, pp.926-929, 2015.

[14] R. Hamamoto, C. Takano, H. Obata, and K. Ishida, "An acceleration of throughput prediction method for access point in multirate wireless LAN considering terminal distribution," IEICE Trans. Fundamentals (Japanese Edition), vol.J98-A, no.2, pp.209-220, Feb. 2015.

[15] S. Biaz and S. Wu, "Rate adaptation algorithms for IEEE 802.11 networks: A survey and comparison," Proc. ISCC 2008, pp.130-136, 2008.

[16] A. Kamerman and L. Monteban, "WaveLAN@-II: A highperformance wireless LAN for the unlicensed band," Bell Labs Technical Journal, vol.2, no.3, pp.118-133, 1997.

[17] H. Suzuki, Y. Kaneko, K. Mase, S. Yamazaki, and H. Makino, "An ad hoc network in the sky, SKYMESH, for large-scale disaster recovery," Proc. 64th IEEE VTC Fall, pp.1-5, 2006.

[18] M. Ishizuka and M. Aida, "The reliability performance of wireless sensor networks configured by power-law and other forms of stochastic node placement," IEICE Trans. Commun., vol.E87-B, no.9, pp.2511-2520, Sept. 2004.

[19] Y. Zou and K. Chakrabarty, "Uncertainty-aware and coverageoriented deployment for sensor networks," J. Parallel Distrib. Comput., vol.64, no.7, pp.788-798, 2004.

[20] N.L. Johnson, A.W. Kemp, and S. Kotz, Univariate discrete distributions, Wiley Series in Probability and Statistics, 2005.

[21] R. Hamamoto, C. Takano, H. Obata, M. Aida, and K. Ishida, "Setting radio transmission range using target problem to improve communication reachability and power saving," Proc. 7th EAI International Conference on ADHOCNETS 2015, Lecture Notes of the Institute for Computer Sciences, Social Informatics and Telecommunications Engineering, vol.155, pp.15-28, Springer, 2015.

[22] IEEE 802.11g, "Further higher data rate extension in the $2.4 \mathrm{GHz}$ band," 2003.

[23] Y. Bejerano, H.-G. Choi, S.-J. Han, and T. Nandagopal, "Performance tuning of infrastructure-mode wireless LANs" Proc. 8th International Symposium on WiOpt 2010, pp.242-251, 2010.

[24] W. Stevens, TCP/IP illustrated, vol.1 the protocols, Addison-Wesley, 1994.

[25] J. Padhye, V. Firoiu, D. Towsley, and J. Kurose, "Modeling TCP throughput: A simple model and its empirical validation," Proc. ACM SIGCOMM'98, pp.303-314, 1998.

[26] G. Bianchi, "Performance analysis of the IEEE 802.11 distributed coordination function," IEEE J. Sel. Areas Commun., vol.18, no.3, pp.535-547, 2000.

[27] H.L. Vu and T. Sakurai, "Collision probability in saturated IEEE 
802.11 networks," Proc. ATNAC 2006, 2006.

[28] NTT Access Network Service Systems Laboratories, ANSL R\&D Times, vol.47, Available at http://www.ansl.ntt.co.jp/j/times/047/01/ 02.html, 2006 (in Japanese).

[29] BUFFALO wireless LAN access point: WAPS-APG600H manual, Available at http://manual.buffalo.jp/buf-doc/35020037-06.pdf, 2015 (in Japanese).

[30] K. Thompson, G.J. Miller, and R. Wilder, "Wide-area Internet traffic patterns and characteristics." IEEE Netw., vol.11, no.6, pp.10-23, 1997.

[31] J.-S. Park, J.-Y. Lee, and S.-B. Lee, "Internet traffic measurement and analysis in a high speed network environment: Workload and flow characteristics," J. Commun. Netw., vol.2, no.3, pp.287-296, 2000.

[32] T. Mori, M. Uchida, and S. Goto, "On the flow analysis of the Internet traffic: Web vs. P2P," IEICE Trans. Inf. \& Syst. (Japanese Edition), vol.J87-D-I, no.5, pp.561-571, May 2004.

[33] F. Maguolo, M. Lacage, and T. Turletti, "Efficient collision detection for auto rate fallback algorithm," Proc. IEEE ISCC 2008, pp.25-30, 2008.

[34] Y. Xi, B.S. Kim, J.B. Wei, and Q.Y. Huang, "Adaptive multirate auto rate fallback protocol for IEEE 802.11 WLANS," Proc. IEEE Military Communications Conference, pp.1-7, 2006.

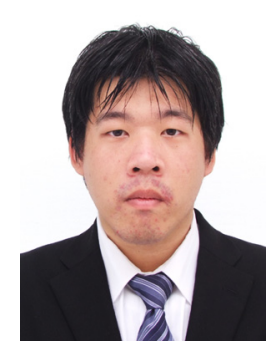

Ryo Hamamoto received B.E., M.E., and Ph.D. degrees in Computer Engineering from Hiroshima City University, Japan, in 2012, 2014, and 2016, respectively. Currently, he is working with Security Research Laboratories, NEC Corporation, Japan. His research interests are wireless networks and distributed systems based on mathematical modeling and analysis. He received the IEICE Information Network Research Award in 2012 and 2015. He is a member of IEEE (U.S.A.).

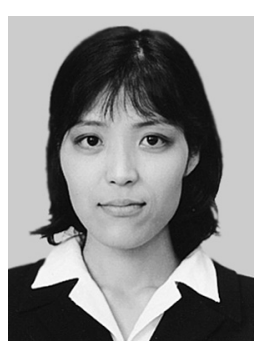

Chisa Takano received a B.E. degree in Telecommunication Engineering from Osaka University, Japan, in 2000, and a Ph.D. in System Design Engineering from Tokyo Metropolitan University, Japan, in 2008. In 2000, she joined the Traffic Research Center, NTT Advanced Technology Corporation. Since April 2008, she has been an Associate Professor at the Graduate School of Information Sciences, Hiroshima City University. Her research interests are computer networks and distributed systems. She received the IEICE Young Researchers' Award in 2003. She received the Information Network Research Award from the IEICE in 2004, 2012, and 2015. She is a member of IEEE (U.S.A.) and IPSJ (Japan).

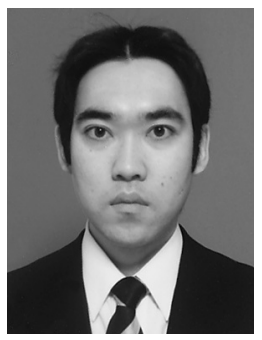

Hiroyasu Obata received B.E., M.E., and Ph.D. degrees in Computer Engineering from Hiroshima City University, Japan, in 2000, 2002, and 2007, respectively. From 2002 to 2003, he was with KDDI Co., Ltd. From 2003 to 2009, he was a Research Associate at Hiroshima City University. From 2009 to 2016, he was a Lecturer at Hiroshima City University. He is currently an Associate Professor in the Graduate School of Information Sciences, Hiroshima City University. His interests include computer communications on wireless networks such as satellite links and wireless LANs, access control, and high speed transport protocols. He received the Information Network Research Award of IEICE in 2015. Dr. Obata is a member of IEEE (U.S.A) and IPSJ (Japan).

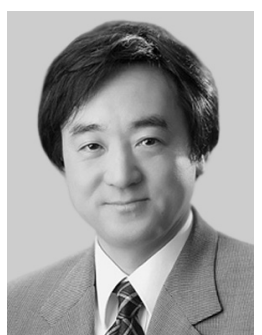

Kenji Ishida received B.E., M.Sc., and Ph.D. degrees from Hiroshima University, Japan, in 1984, 1986, and 1989, respectively. He was at Hiroshima Prefectural University from 1989 to 1997. From 1997 to 2003, he was an Associate Professor at Hiroshima City University. Since 2003, he has been a Professor in the Department of Computer Engineering, Faculty of Information Sciences, Hiroshima City University. His interests include distributed computing systems and designing control procedures for computer networks. He received the Information Network Research Award of IEICE in 1998, 2000, 2012, and 2015. Dr. Ishida is a member of IEEE (U.S.A.), ACM (U.S.A.), and IPSJ (Japan). 